\title{
Inter-laminar microcircuits across neocortex: repair and augmentation
}

\author{
Ioan Opris* \\ Department of Physiology and Pharmacology, Wake Forest University School of Medicine, Winston-Salem, NC, USA \\ *Correspondence: ioopris@wfubmc.edu \\ Edited by: \\ Mikhail Lebedev, Duke University, USA \\ Reviewed by: \\ Manuel Casanova, University of Louisville, USA
}

Keywords: cortical minicolumn, cortical layer, cortical module, microcircuit, neocortex, repair, brain machine interface, prosthetics

\section{INTRODUCTION}

Repair and brain augmentation approaches, such as brain-machine interfaces, neural stimulation and other neural prostheses, have experienced a rapid development during the last decade (Nicolelis et al., 2003; Lebedev and Nicolelis, 2006). Still, only few of these methods target the fine microcircuitry of the brain (Jones and Rakic, 2010; Opris et al., 2012a). Here, it is highlighted the potential employing of inter-laminar recording and microstimulation of cortical microcircuits to build neural prostheses for repair and augmentation of cognitive function. In the future, such microcircuit-based prostheses will provide efficient therapies for patients with neurological and psychiatric disorders. Moreover, it is implied that neural enhancement approaches can be applied to inter-laminar microcircuits across the entire cortex.

\section{CORTICAL MICROCIRCUITS}

As proposed by Mountcastle, the primate neocortical circuitry has a modular architecture that subserves a multitude of sensory (visual, auditory, touch), motor, cognitive (attention, memory, decision) and emotional functions (Mountcastle, 1957, 1997; Opris and Bruce, 2005; Shepherd and Grillner, 2010). These modules are composed of elementary building blocks formed by vertical arrangements of cortical neurons, called minicolumns (Szentágothai and Arbib, 1975; Mountcastle, 1997). Within minicolumns, cortical neurons are aggregated into six horizontal layers (or laminae): three supra-granular layers (L1-L3), a granular layer (L4) and two infra-granular layers (L5/L6) (Figure 1A). The granular layer receives sensory input from thalamus (Constantinople and Bruno, 2013). The supra-granular layers consist of small pyramidal neurons that form a complex network of intra-cortical connections, particularly the connections to the infra-granular layers of larger pyramidal neurons that generate most of the output from cerebral cortex to other parts of the brain (Buxhoeveden and Casanova, 2002). According to this three stratum functional module, infra-granular layers execute the associative computations elaborated in supra-granular layers (Buxhoeveden and Casanova, 2002; Casanova et al., 2011).

Here, the focus is on inter-laminar cortical microcircuits formed by interconnected pyramidal neurons from the supra-granular and infra-granular layers (Thomson and Bannister, 2003; Opris et al., 2011, 2012a,b, 2013). These microcircuits receive input from neurons in layer L4, which project to $\mathrm{L} 2 / 3$, or through direct thalamic projections to the supragranular layers in the higher-order cortical areas. Neurons in L2/3 then project topdown to L5, where they target specific types of pyramidal cells and inhibitory interneurons. Some L5 neurons project back to L2/3 neurons, forming an interlaminar loop (Weiler et al., 2008) or back to L4, targeting mostly interneurons (Thomson and Bannister, 2003). The outputs from cortical microcircuits, corticostriatal projections arise mostly from L5, whereas cortico-thalamic projections arise from L6.

Cortical microcircuits are strikingly similar across the neocortex (hence the term "canonical microcircuits"). It has been suggested that such repeatability in the microcircuit pattern plays a key role in reducing the errors of encoding (Bastos et al., 2012). Some characteristics of microcolumns are specific to particular cortical areas. For example, the thickness of L4 is different across areas (DeFelipe et al., 2012). It is most prominent in sensory areas and the thinnest in the motor cortex. There are also area-specific differences in the topographic connectivity of microcircuits with their cortical and subcortical projection areas (Das and Gilbert, 1995; Kritzer and Goldman-Rakic, 1995; Opris et al., 2013).

\section{INTER-AREA CONNECTIVITY}

Cortical microcircuits are connected into a macro-network by cortico-cortical connections, which link areas within the same hemisphere, as well as between hemispheres (Van Essen et al., 1982). This super network subserves the "perceptionto-action" cycle-a group of processes that handle environmental stimuli and convert them into actions (Romo et al., 2002; Fuster and Bressler, 2012). Microcircuits within the same hemisphere are interconnected (from low level sensory to high level associative processes) through horizontal connections in lamina $2 / 3$, spanning over many cortical areas (Das and Gilbert, 1995; Kritzer and Goldman-Rakic, 1995; Fuster and Bressler, 2012).

Inter-area connectivity of cortical microcircuits preserves spatial topography suggesting a column-to-column match from one area to another (e.g., Figure 1B schematics of V1 projections to prefrontal area 46 through the dorsal visual stream; Goldman-Rakic, 1996). Additionally, the topography is preserved within minicolumns owing to the interlaminar projections (Opris et al., 2013). Interhemispheric connectivity is formed by neural interconnections of lamina $3 \mathrm{~b}$ (Jones et al., 1979; Van Essen et al., 1982). 


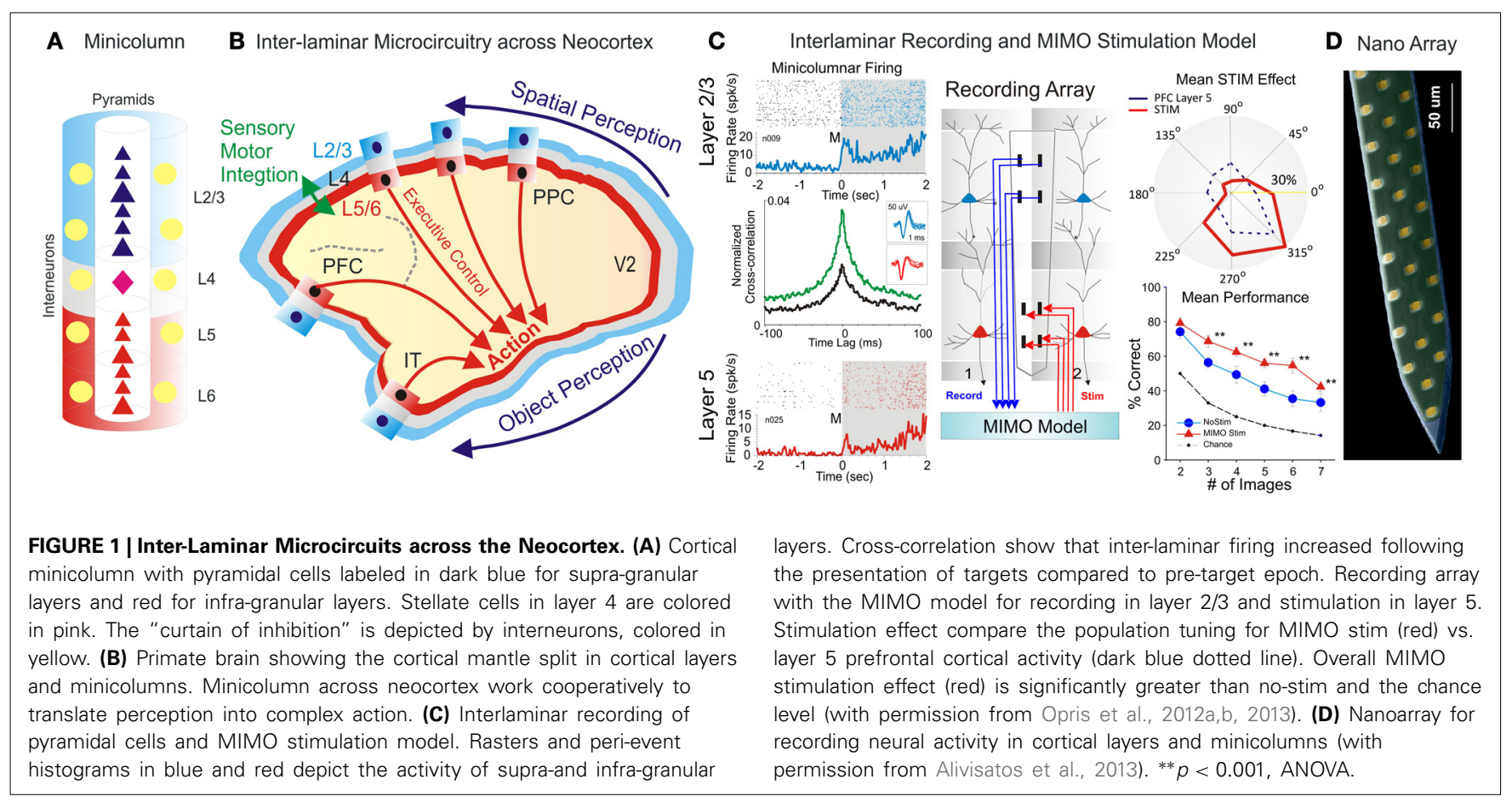

\section{MICROCIRCUITS AND COGNITION}

Recent research conducted in non-human primates indicates that a variety of sensory, motor and executive functions emerge from the interactions between frontal, parietal, temporal and occipital cortical microcircuits (Atencio and Schreiner, 2010; Buffalo et al., 2011; Takeuchi et al., 2011; Hansen et al., 2012; Opris et al., 2012a,b, 2013; Hirabayashi et al., 2013a,b; Mahan and Georgopoulos, 2013). Moreover, several augmentation approaches based on microcircuits have been implemented. These advances have been possible owing to the development of new multi-electrode arrays (MEA) fitted for recordings from neural elements of cortical columns (Moxon et al., 2004). Thus, MEAs with linear or bi-linear geometry have been successfully employed for simultaneous recordings from supra- and infragranular cortical laminae in adjacent minicolumns, resulting in unprecedented insights into the function of cortical microcircuits (Mo et al., 2011; Opris et al., 2011, 2012a,b, 2013).

A number of recent publications suggest that cortical microcircuits perform elementary computations while cognitive functions are sub-served by a broader network comprising multiple cortical areas (Fuster and Bressler, 2012). For example, elementary computations related to executive control are performed by microcircuits in the prefrontal cortex (Opris et al., 2012a,b), whereas microcircuits of the temporal cortex maintain long term memory (Takeuchi et al., 2011; Hirabayashi et al., 2013a). Prefrontal microcircuits are in a unique and privileged position at the top of sensory-to-motor hierarchy network because they coordinate a multitude of stimuli, perceptions, biases and actions related to such functions as attention, decision making, and working memory. As such, prefrontal microcicuits integrate and synthetize signals over a broad spectrum of perceptual stimuli and various modalities. This integration is performed in supragranular layers, whereas the output of the infra-granular layers provides selectionrelated signals, which are sent back to the infra-granular layers and the other areas comprising the network. As a matter of fact, signals can reverberate within interlaminar loops. Thus, cortical microcircuits for long term memory in entorhinal cortex and hippocampal formation employ such reverberating signals (Takeuchi et al., 2011) to integrate relevant information over time (Fuster, 2001).

Our group at Wake Forest University in collaboration with Dr. Berger's team at USC and Dr. Gerhard's group at University of Kentucky, examined the executive function of prefrontal microcircuits (Opris et al., 2012a,b, 2013). We trained rhesus monkeys to select a target (spatial or object) for hand movement, after a memory delay, while the neural activity in prefrontal microcircuits was recorded (Figure 1C). Our electrode arrays were specifically designed to record from neurons located in both supra- \& infragranular layers of adjacent minicolumns. We analyzed correlated firing in neurons from the supra- and infra-granular layers. Interestingly, the extent of correlated firing was linked to the accuracy of monkey performance. Correlated firing between cell pairs within single minicolumns was higher during correct selections and reduced in error trials (Opris et al., 2012a). Thus, we discovered that animals make errors when their prefrontal cortical microcircuits do not function properly when handle task relevant information. Additionally, we discovered that during the presentation of the target and during the executive selection of the correct target, assemblies of cell firing in prefrontal layers exhibited similar tuning to target locations on behavioral trials in which this information was important. These studies provided a direct demonstration of real-time 
inter-laminar processing of information in prefrontal microcircuits during decisionmaking (Opris and Bruce, 2005; Opris et al., 2012a).

\section{COGNITIVE ENHANCEMENT APPROACHES BASED ON MICROCIRCUITS}

Recent studies have demonstrated that cognitive enhancement can be achieved by microstimulation of specific elements of cortical microcircuits (Opris et al., 2001, 2013; Hampson et al., 2012). These enhancement methods employed a multiinput/multi-output (MIMO) Volterra kernel-based non-linear dynamic model, which was applied to the spatiotemporal patterns of neuronal firing recorded in prefrontal cortical layers L2/3 and L5 to convert the firing of neurons in layer $2 / 3$ into microstimulation patterns applied to layer 5 (Berger et al., 2011; Hampson et al., 2012). MIMO model is based on the principle of multiplexing, where a high rate signal is split into several low rate signals, which are then sent to multiple recipients via multiple channels. Using multiple channels of information transfer MIMO model provides a more reliable communication (Figure 1C, right panel).

To perform cognitive augmentation, inter-laminar recordings are analyzed via a non-linear MIMO model, whose output is then converted into patterns of microstimulation (Berger et al., 2011). In these studies, MIMO models used a precise topographically matched stimulation by extracting the patterns of firing that relate to the successful behavioral performance. This allowed the substitution of task-related laminar L5 neuron firing patterns with electrical stimulation in the same recording regions during columnar transmission from lamina L2/3 at the time of target selection. Such stimulation improved normal task performance, but more importantly, recovered performance after being impaired by a pharmacological disruption of decision making (Hampson et al., 2012). Moreover, the fact that stimulation-induced spatial preference (in percent correct performance) on spatial trials that was similar to neural tuning indicated that inter-laminar prefrontal microcircuits played causal roles to the executive function (Opris et al., 2005, 2013). These findings provided the first successful demonstration of a microcircuit-based neuroprosthesis designed specifically to restore or repair disrupted cognitive function.

\section{NEUROLOGICAL DISEASES AND MICROCIRCUITS}

Disruption of inter-laminar microcircuits within cortical minicolums is a signature of a broad spectrum of neurological and psychiatric disorders, such as autism (Casanova, 2013), schizophrenia (Di Rosa et al., 2009), Alzheimer's disease (Chance et al., 2011) drug addiction (Opris et al., 2012a) and other disorders. The use of both invasive MIMO stimulation (Hampson et al., 2012) and non-invasive transcranial magnrtic stimulation (TMS; Sokhadze et al., 2012) are valuable potential options to repair or treat such dysfunctions. The multitude of deficits in a cortical microcircuit involve the micro-anatomic disconnections between layers or within minicolumns (autism, schizophrenia, Alzheimer), the intra- and inter-laminar neuromodulation (drug addiction, aging), the lack or excess of inhibition (ADHD, depression), etc.

Microcircuit-based neuroprostheses, such as MIMO based memory implants (Berger et al., 2011), and decision chips (Hampson et al., 2012) hold the promise to provide treatment for neurological conditions that result from compromised microcircuits. Targeting cortical microcircuitry may be key to the development of next-generation enhancement methods and medical treatments.

\section{FUTURE DIRECTIONS FOR MICROCIRCUIT-BASED APPROACHES}

An emerging approach with broad implications for basic and clinical neuroscience is based on optogenetic stimulation (Gradinaru et al., 2007; Tye and Deisseroth, 2012). Recent developments in optogenetics based on optical manipulation of activity in neural circuits with light-sensitive rhodopsins, such as the Chlamydomonas channelrhodopsin-2 (ChR2) are now capable to stimulate the inter-laminar microcircuits at millisecond-scale, with cell type-specific effects of optical perturbations in nonhuman primates (Diester et al., 2011; Han,
2012), opening up new possibilities for repair and augmentation.

Recent developments in nanotechnological tools and in the design and synthesis of nano-materials have generated optical, electrical, and chemical methods that can readily be adapted for use in neuroscience. Nanotechnology was instrumental to nanofabricated planar electrode array (Figure 1D) for high-density neuronal voltage recording (Du et al., 2011; Suyatin et al., 2013). Leveraging microand nanofabrication technology raises the prospect for creating vastly greater numbers of electrodes and smaller, less invasive implantable devices. A promising category for brain microcircuits is the planar electrode array (Viventi et al., 2011; Alivisatos et al., 2013), which is patterned on a crystalline, ceramic, or polymer support structure (Figure 1D). The recording of neuronal activity with threedimensional (3D) microelectrode arrays (Zorzos et al., 2012) represents a major advance in brain activity mapping techniques, by providing a tool to probe how intra and inter-laminar/regional neural circuits cooperate to process information. Building prosthetic minicolumns as basic modules to repair the damaged cortical tissue will become a valuable approach in the cognitive neuroprosthetics.

To trace the flow of neural signals in the cortical microcircuits across neocortex, or in the large scale brain networks, analytical tools based on dynamic Bayesian networks and Granger causality are available (Granger, 1969; Smith et al., 2006). These methods allow to identify putative causal interactions and population codes within the neural circuits involved in perception and behavior (Yu et al., 2004; Beck et al., 2008).

Microcircuit-based augmentation could be implemented in several cortical areas, where different functions could be enhanced. Thus, the prefrontal cortical microcircuits involved in attention, working memory, executive decisions and conflict monitoring may be augmented for autism (Casanova et al., 2010), schizophrenia (Chance et al., 2011), drug addiction (Opris et al., 2012a), Alzheimer's or attention deficit disorders.

In conclusion, a better understanding of the function of inter-laminar microcircuits across the neocortex is needed for the 
development of treatments for neurological disorders, as well as for the development of methods of brain augmentation.

\section{ACKNOWLEDGMENTS}

The author would like to thank Drs. Samuel A. Deadwyler, Mikhail A. Lebedev and Manuel F. Casanova for reading the manuscript and for the valuable insights provided.

\section{REFERENCES}

Alivisatos, A. P., Andrews, A. M., Boyden, E. S., Chun, M., Church, G. M., Deisseroth, K., et al. (2013). Nanotools for neuroscience and brain activity mapping. Nano Focus 7, 1850-1866. doi: $10.1021 / \mathrm{nn} 4012847$

Atencio, C. A., and Schreiner, C. E. (2010). Columnar connectivity and laminar processing in cat primary auditory cortex. PLOS ONE 5:e9521. doi: 10.1371/journal.pone.0009521

Bastos, A. M., Usrey, W. M., Adams, R. A., Mangun, G. R., Fries, P., and Friston, K. J. (2012). Canonical microcircuits for predictive coding. Neuron 76, 695-711. doi: 10.1016/j.neuron.2012.10.038

Beck, J. M., Ma, W. J., Kiani, R., Hanks, T., Churchland, A. K., Roitman, J., et al. (2008). Probabilistic population codes for Bayesian decision making. Neuron 60, 1142-1152. doi: 10.1016/j.neuron.2008.09.021

Berger, T. W., Hampson, R. E., Song, D., Goonawardena, A., Marmarelis, V. Z., and Deadwyler, S. A. (2011). A cortical neural prosthesis for restoring and enhancing memory. $J$. Neural Eng. 8, 046017. doi: 10.1088/1741-2560/8/ 4/046017

Buffalo, E. A., Fries, P., Landman, R., Buschman, T. J., and Desimone, R. (2011). Laminar differences in gamma and alpha coherence in the ventral stream. Proc. Natl. Acad. Sci. U.S.A. 108, 11262-11267. doi: 10.1073/pnas.1011284108

Buxhoeveden, D. P., and Casanova, M. F. (2002). The minicolumn hypothesis in neuroscience. Brain 125, 935-951. doi: 10.1093/brain/awf110

Casanova, M. F. (2013). Neural Mechanisms in Autism, Encyclopedia of autism spectrum disorders. Heidelberg: Springer.

Casanova, M. F., Ayman El-Baz, A., Vanbogaert, E., Narahari, P., and Switala, A. (2010). A topographic study of minicolumnar core width by lamina comparison between autistic subjects and controls: Possible minicolumnar disruption due to an anatomical element in common to multiple laminae. Brain Pathol. 20, 451-458. doi: 10.1111/j.1750-3639.2009.00319.x

Casanova, M. F., Ayman El-Baz., and Switala, A. E. (2011). Laws of conservation as related to brain growth, aging, and evolution: symmetry of the minicolumn. Front. Neuroanat. 5:66. doi: 10.3389/fnana.2011.00066

Chance, S. A., Clover, L., Cousijn, H., Currah, L., Pettingill, R., and Esiri, M. M. (2011). Microanatomical correlates of cognitive ability and decline: normal ageing, MCI, and Alzheimer's disease. Cereb. Cortex 21, 1870-1878. doi: 10.1093/ cercor/bhq264
Constantinople, C. M., and Bruno, R. M. (2013). Deep cortical layers are activated directly from thalamus. Science 340, 1591-1594. doi: $10.1126 /$ science. 1236425

Das, A., and Gilbert, C. D. (1995). Long-range horizontal connections and their role in cortical reorganization revealed by optical recording of cat primary visual cortex. Nature $375,780-784$. doi: 10.1038/375780a0

DeFelipe, J., Markram, H., and Rockland, K. S. (2012). The neocortical column. Front Neuroanat. 6, 22. doi: 10.3389/fnana.2012.00005

Di Rosa, E., Crow, T. J., Walker, M. A., Black, G., and Chance, S. A. (2009). Reduced neuron density, enlarged minicolumn spacing and altered ageing effects in fusiform cortex in schizophrenia. Psychiatry Res. 166, 102-115. doi: 10.1016/j.psychres.2008.04.007

Diester, I., Kaufman, M. T., Mogri, M., Pashaie, R., Goo, W., Yizhar, O., et al. (2011). An optogenetic toolbox designed for primates. Nat. Neurosci. 14, 387-397. doi: 10.1038/nn.2749

Du, J., Blanche, T. J., Harrison, R. R., Lester, H. A., and Masmanidis, S. C. (2011). Multiplexed high density electrophysiology with nanofabricated neural probes. PLoS ONE 6:e26204. doi: 10.1371/journal.pone.0026204

Fuster, J. M. (2001). The prefrontal cortex-an update: time is of the essence. Neuron 2, 319-333. doi: $10.1016 / \mathrm{S} 0896-6273(01) 00285-9$

Fuster, J. M., and Bressler, S. L. (2012). Cognit activation: a mechanism enabling temporal integration in working memory. Trends Cogn. Sci. 16, 207-218. doi: 10.1016/j.tics.2012.03.005

Goldman-Rakic, P. S. (1996). The prefrontal landscape: implications of functional architecture for understanding human mentation and the central executive. Philos. Trans. $R$ Soc. Lond. B Biol. Sci. 351, 1445-1453. doi: 10.1098/rstb. 1996.0129

Gradinaru, V., Thompson, K. R., Zhang, F., Mogri, M., Kay, K., Schneider, M. B., et al. (2007). Targeting and readout strategies for fast optical neural control in vitro and in vivo. J. Neurosci. 27, 14231-14238. doi: 10.1523/JNEUROSCI.357807.2007

Granger, C. W. J. (1969). Investigating causal relations by econometric models and crossspectral methods. Econometrica 37, 424-438. doi: $10.2307 / 1912791$

Hampson, R. E., Gerhardt, G. A., Marmarelis, V., Song, D., Opris, I., Santos, L., et al. (2012). Facilitation and restoration of cognitive function in primate prefrontal cortex by a neuroprosthesis that utilizes minicolumn-specific neural firing. J. Neural Eng. 9:056012. doi: 10.1088/17412560/9/5/056012

Han, X. (2012). In vivo application of optogenetics for neural circuit analysis. ACS Chem. Neurosci. 3, 577-584. doi: 10.1021/cn300065j

Hansen, B. J., Chelaru, M. I., and Dragoi, V. (2012). Correlated variability in laminar cortical circuits. Neuron. 76, 590-602. doi: 10.1016/j.neuron.2012.08.029

Hirabayashi, T., Takeuchi, D., Tamura, K., and Miyashita, Y. (2013a). Microcircuits for hierarchical elaboration of object coding across primate temporal areas. Science. 341, 191-195. doi: 10.1126/science. 1236927
Hirabayashi, T., Takeuchi, D., Tamura, K., and Miyashita, Y. (2013b). Functional microcircuit recruited during retrieval of object association memory in monkey perirhinal cortex. Neuron 77, 192-203. doi: 10.1016/j.neuron.2012.10.03

Jones, E. G., Coulter, J. D., and Wise, S. P. (1979). Commissural columns in the sensory-motor cortex of monkeys. J. Comp. Neurol. 188, 113-135. doi: $10.1002 / \mathrm{cne} .901880110$

Jones, E. G., and Rakic, P. (2010). Radial columns in cortical architecture: it is the composition that counts. Cereb. Cortex 20, 2261-2264. doi: 10.1093/cercor/bhq127

Kritzer, M. F., and Goldman-Rakic, P. S. (1995). Intrinsic circuit organization of the major layers and sublayers of the dorsolateral prefrontal cortex in the rhesus monkey. J. Comp. Neurol. 359, 131-143. doi: 10.1002/cne.903590109

Lebedev, M. A., and Nicolelis, M. A. L. (2006). Brain-machine interfaces: past, present and future. Trends Neurosci. 29, 536-546. doi: 10.1016/j.tins.2006.07.004

Mahan, M. Y., and Georgopoulos, A. P. (2013). Motor directional tuning across brain areas: directional resonance and the role of inhibition for directional accuracy. Front. Neural. Circuits 7:92. doi: 10.3389/fncir.2013.00092

Mo, J., Schroeder, C. E., and Ding, M. (2011). Attentional modulation of alpha oscillations in macaque inferotemporal cortex. J. Neurosci. 31, 878-882. doi: 10.1523/JNEUROSCI.5295-10.2011

Mountcastle, V. B. (1957). Modality and topographic properties of single neurons of cat's somatic sensory cortex. J. Neurophysiol. 20, 408-434.

Mountcastle, V. B. (1997). The columnar organization of the neocortex. Brain 120, 701-722. doi: 10.1093/brain/120.4.701

Moxon, K. A., Leiser, S. C., Gerhardt, G. A., Barbee, K. A., and Chapin, J. K. (2004). Ceramic-based multisite electrode arrays for chronic single-neuron recording. IEEE Trans. Biomed. Eng. 51, 647-656. doi: 10.1109/TBME.2003.821037

Nicolelis, M. A., Dimitrov, D., Carmena, J. M., Crist, R., Lehew, G., Kralik, J. D., et al. (2003). Chronic, multisite, multielectrode recordings in macaque monkeys. Proc. Natl. Acad. Sci. U.S.A. 100, 11041-11046. doi: 10.1073/pnas. 1934665100

Opris, I., Barborica, A., and Ferrera, V. P. (2001). A gap effect during microstimulation in the prefrontal cortex of monkey. Exp. Brain Res. 138, 1-7. doi: $10.1007 /$ s002210100686

Opris, I., Barborica, A., and Ferrera, V. P. (2005). Microstimulation of dorsolateral prefrontal cortex biases saccade target selection. J. Cogn. Neurosci. 17, 893-904. doi: 10.1162/0898929054021120

Opris, I., and Bruce, C. J. (2005). Neural circuitry of judgment and decision mechanisms. Brain Res. Rev. 48, 509-528. doi: 10.1016/j.brainresrev.2004.11.001

Opris, I., Hampson, R. E., Gerhardt, G. A., Berger, T. W., and Deadwyler, S. A. (2012a). Columnar processing in primate pFC: evidence for executive control microcircuits. J. Cogn. Neurosci. 24 2334-2347. doi: 10.1162/jocn_a_00307

Opris, I., Fuqua, J. L., Huettl, P. F., Gerhardt, G. A., Berger, T. W., Hampson, R. E., et al. (2012b). Closing the loop in primate prefrontal cortex: inter-laminar processing. Front. Neural Circuits 6:88. doi: 10.3389/fncir.2012.00088 
Opris, I., Hampson, R. E., Stanford, T. R., Gerhardt, G. A., and Deadwyler, S. A. (2011). Neural activity in frontal cortical cell layers: evidence for columnar sensorimotor processing. J. Cogn. Neurosci. 23, 1507-1521. doi: 10.1162/jocn.2010.21534

Opris, I., Santos, L. M., Song, D., Gerhardt, G. A., Berger, T. W., Hampson, R. E., et al. (2013). Prefrontal cortical microcircuits bind perception to executive control. Sci. Rep. 3:2285. doi: 10.1038/srep02285

Romo, R., Hernández, A., Salinas, E., Brody, C. D., Zainos, A., Lemus, L., et al. (2002). From sensation to action. Behav. Brain Res. 135, 105-118. doi: 10.1016/S0166-4328(02)00161-4

Shepherd, G., and Grillner, S. (2010). Handbook of Brain Microcircuits. Oxford: Oxford University Press.

Smith, V. A., Yu, J., Smulders, T. V., Hartemink, A. J., and Jarvis, E. D. (2006). Computational inference of neural information flow networks. PLoS Comput. Biol. 2:e161. doi: 10.1371/journal.pcbi.0020161

Sokhadze, E. M., Baruth, J. M., Sears, L., Sokhadze, G. E., El-Baz, A. S., and Casanova, M. F. (2012). Prefrontal neuromodulation using rTMS improves error monitoring and correction function in autism. Appl. Psychophysiol. Biofeedback 37, 91-102. doi: 10.1007/s10484-012-9182-5

Suyatin, D. B., Wallman, L., Thelin, J., Prinz, C. N., Jorntell, H., Samuelson L., et al. (2013).
Nanowire-based electrode for acute in vivo neural recordings in the brain. PLOS ONE 8:e56673. doi: 10.1371/journal.pone. 0056673

Szentágothai, J., and Arbib, M. A. (1975). Conceptual Models of Neural Organization. Cambridge, MA: MIT Press.

Takeuchi, D., Hirabayashi, T., Tamura, K., and Miyashita, Y. (2011). Reversal of interlaminar signal between sensory and memory processing in monkey temporal cortex. Science 331, 1443-1447. doi: 10.1126/science.1199967

Thomson, A. M., and Bannister, A. P. (2003). Interlaminar connections in the neocortex. Cereb. Cortex 13, 5-14. doi: 10.1093/cercor/ 13.1.5

Tye, K. M., and Deisseroth, K. (2012). Optogenetic investigation of neural circuits underlying brain disease in animal models. Nat. Rev. Neurosci. 13, 251-266. doi: 10.1038/nrn3171

Van Essen, D., Newsome, W. T., and Bixby, J. L. (1982) The pattern of interhemispheric connections and its relationship to the extrastriate visual area in the macaque monkey. J. Neurosci. 2, 265-283.

Viventi, J., Kim, D.-H., Vigeland, L., Frechette, E. S., Blanco, J. A., Kim, Y.-S., et al. (2011). Flexible, foldable, actively multiplexed, high-density electrode array for mapping brain activity in vivo. Nat. Neurosci. 14, 1599-1605. doi: 10.1038/nn.2973

Weiler, N., Wood, L., Yu, J., Solla, S. A., and Shepherd, G. M. (2008). Top-down laminar organization of the excitatory network in motor cortex. Nat. Neurosci. 11, 360-366. doi: 10.1038/nn2049

Yu, J., Smith, V. A., Wang, P. P., Hartemink, A. J., and Jarvis, E. D. (2004). Advances to Bayesian network inference for generating causal networks from observational biological data. Bioinformatics 20, 3594-3603. doi: 10.1093/bioinformatics/bth448

Zorzos, A. N., Scholvin, J., Boyden, E. S., and Fonstad, C. G. (2012). Three-dimensional multiwaveguide probe array for light delivery to distributed brain circuits. Opt. Lett. 37, 4841-4843. doi: 10.1364/OL.37.004841

Received: 12 October 2013; accepted: 19 October 2013; published online: 19 November 2013.

Citation: Opris I (2013) Inter-laminar microcircuits across neocortex: repair and augmentation. Front. Syst. Neurosci. 7:80. doi: 10.3389/fnsys.2013.00080

This article was submitted to the journal Frontiers in Systems Neuroscience.

Copyright (c) 2013 Opris. This is an open-access article distributed under the terms of the Creative Commons Attribution License (CC BY). The use, distribution or reproduction in other forums is permitted, provided the original author(s) or licensor are credited and that the original publication in this journal is cited, in accordance with accepted academic practice. No use, distribution or reproduction is permitted which does not comply with these terms. 